\title{
Disminución del índice de reprobación mediante estrategias tutoriales
}

Alberto Carreón Rodríguez, Sergio Díaz Camacho, Juan Carlos Pérez Merlos y Mireya Salgado Gallegos

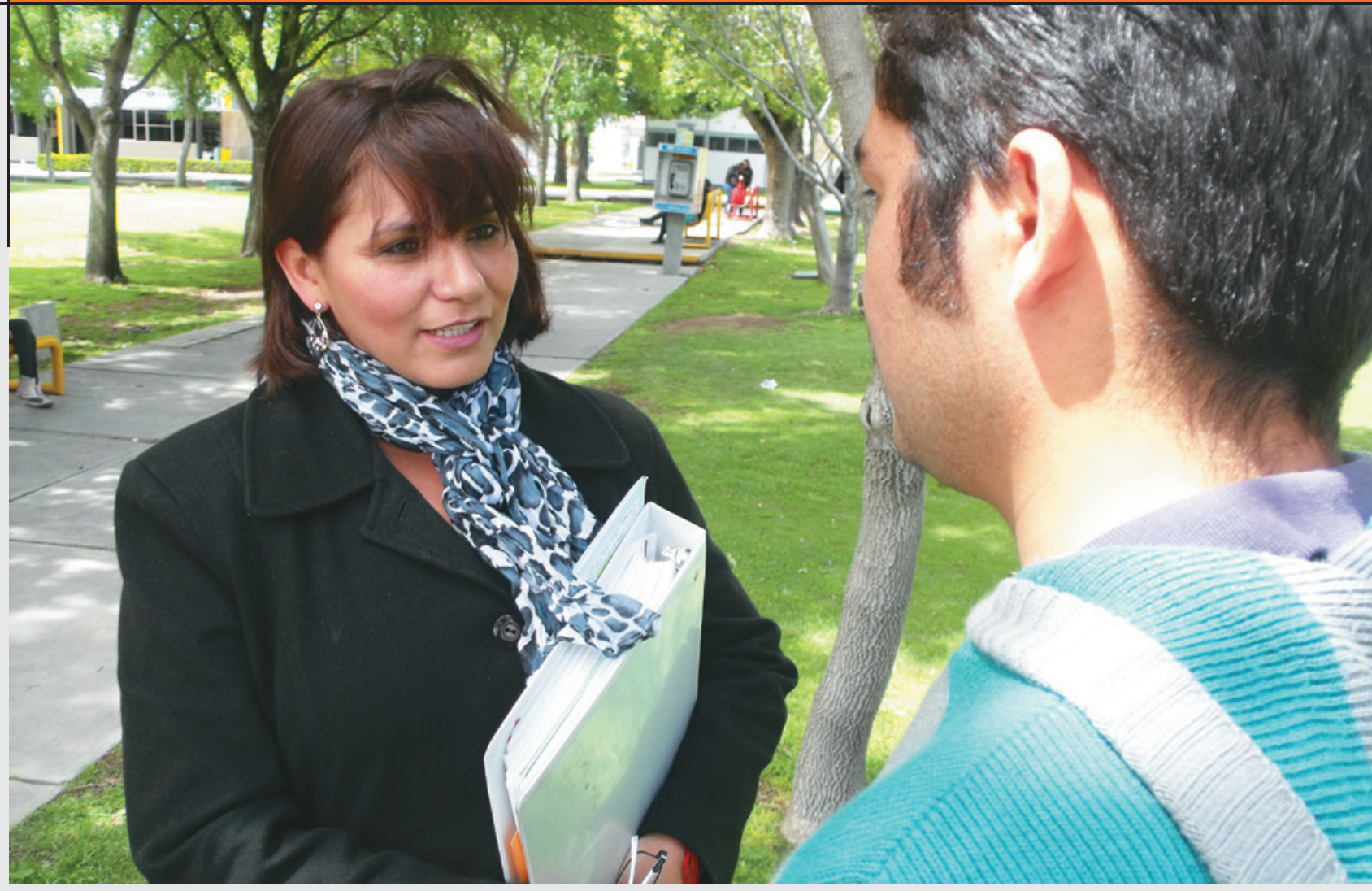

Resumen

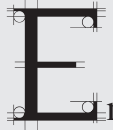

n la Facultad de Ingeniería de la Universidad Autónoma del Estado de México (UAEMex), la asignatura de Cálculo Vectorial, que se imparte en el tercer semestre de las carreras que se ofertan en esa institución y que forma parte del tronco común de cada uno de los planes de estudio, se caracteriza por presentar un alto índice de reprobación. Se sabe bien que esta problemática existe desde el nivel básico hasta el superior, en este último provocando deserción de los estudiantes; por este motivo, se decidió implementar una alternativa de apoyo académico a los alumnos de segundo curso $;^{1}$ se realizó un estudio longitudinal en tiempo, antes y después de aplicar las estrategias tutoriales en un periodo de cuatro años, logrando disminuirlo hasta $20 \%$.

1 El alumno tiene derecho a cursar dos veces la misma materia, cada curso con sus tres evaluaciones: ordinaria, extraordinaria y a título de suficiencia. Segundo curso: significa estar cursando por segunda vez la misma asignatura, en este caso, Cálculo Vectorial. 


\section{Introducción}

A nivel mundial, es conocida la existencia de un alto índice de reprobación en las asignaturas pertenecientes al área de las matemáticas, principalmente en la ingeniería (Camarena, 2008).

Actualmente, la deserción y la reprobación de los estudiantes universitarios de las carreras de ingeniería han pasado a ser, en años recientes, una gran preocupación en las instituciones de educación superior en México. Ocampo et al. (2010) mencionan que el abandono de los estudios universitarios tiene un costo de entre 141 y 415 millones de dólares.

Sobre la referida problemática, Chávez y Zurita (2005), citando a Menéndez en las conclusiones de sus estudios acerca de la reprobación y deserción, afirman que existe una relación significativa entre la reprobación y la deserción escolar. Desde otra perspectiva, Abril Valdez (2008), en un análisis de seguimiento estadístico en Sonora, menciona que no se tienen muchos estudios acerca de la deserción en los diferentes niveles de educación, su trabajo resalta que en el nivel medio superior $49 \%$ de los hombres abandona los estudios y en mujeres $25 \%$, esto debido a la reprobación de materias. Otro estudio realizado en la Facultad de Ingeniería de la Universidad Autónoma de Baja California (Ocampo et al., 2010) hace referencia a que la deserción-reprobación alcanza hasta $60 \%$, y que la deserción en las universidades públicas se calcula en 58\%, mientras que en las privadas en 39\%. Por su parte, De la Cruz et al. (2008) mencionan que en el nivel superior los mayores índices de reprobación se presentan en el área de matemáticas.

Con base en lo anterior, la ANUIES, en el año 2001 (Aparicio, 2004), señala que cada institución educativa debe diseñar estrategias e instrumentar acciones que tengan como propósito incrementar la calidad del proceso formativo integral de los estudiantes, aumentar su rendimiento académico, reducir la reprobación y la deserción escolar, y lograr índices de aprovechamiento y eficiencia terminal satisfactorios.

$\mathrm{Al}$ respecto, en el área de matemáticas, especialmente en Cálculo Integral y Vectorial, Viviana Costa et al. (2010) utilizan un software especializado, como lo es Maple ${ }^{\circledR 2}$ (Maplesoft, 2014), y proponen estrategias de trabajo colaborativo; De la Cruz et al. (2008) describen una estrategia a través de un modelo de diseño didáctico con enfoque cognitivo basado en la resolución de problemas; Prado y González (2003) hacen referencia a un modelo basado en estrategias didácticas y psicológicas en la aplicación de las matemáticas mediante el uso de la computadora, proponen la vinculación de las matemáticas con otras áreas del conocimiento, el trabajo en equipo, enfatizar la modelación matemática y el uso de la tecnología; Montañez et al. (2009) describen el uso del método de Aprendizaje Basado en Proyectos (ABP) para la materia de Cálculo Diferencial, haciendo uso también de software como Matlab ${ }^{\circledR 3}$ (MathWorks, 2014); y Ríos (2009) utiliza preexámenes como estrategia de mejora del aprendizaje en la materia de Física.

2 Programa orientado a la resolución de problemas matemáticos, capaz de realizar cálculos simbólicos, algebraicos y de álgebra computacional.

3 Es un entorno interactivo para el cálculo numérico, la visualización y la programación usando matrices. 
Por lo anterior, se puede observar que en la literatura consultada no se han encontrado trabajos publicados que hagan referencia explícitamente al uso de estrategias para disminuir los índices de reprobación, específicamente relacionadas con el Cálculo Vectorial, materia que se imparte en los primeros semestres del tronco común de las licenciaturas que ofrece la Facultad de Ingeniería de la UAEMex.

Este trabajo integra un análisis de la problemática de reprobación que presenta la materia de Cálculo 3 (Cálculo Vectorial) en esta institución en conjunto con la aplicación de estrategias para disminuir el caso de la reprobación y bajar implícitamente el índice de deserción. 


\section{Desarrollo}

El caso particular de la Facultad de Ingeniería de la UAEMex, en cuanto a reprobación se refiere, tiene sus inicios en los primeros semestres debido a las carencias, dificultades y errores en los conocimientos matemáticos básicos con los que los alumnos cuentan al ingresar a un plan de estudios, lo cual ha ocasionado que materias como Geometría Analítica, Cálculo 1, Cálculo 2 y Cálculo 3 sean las que presentan un mayor índice de reprobación y rezago, además de que presentan incidencias específicas en la trayectoria académica de los alumnos.

Una de las incidencias que más presentan los alumnos en las asignaturas antes mencionadas es lo que se denomina segundo curso, es decir, los estudiantes inciden en cursar por dos ocasiones una materia, llegando en ocasiones a presentar baja definitiva del programa de estudios cuando éste no llega a aprobar la materia por segunda ocasión en ninguna de sus tres oportunidades de evaluación (ordinario, extraordinario y título de suficiencia).

Ante esta problemática, como primera etapa, se trabajó con la materia de Cálculo Vectorial, principalmente con los alumnos de segundo curso, ya que éstos presentaron los menores índices de acreditación durante el periodo 2010A (febrero-julio 2010) al 2011B (agosto 2011-enero 2012).

A partir del semestre 2012A, a la fecha, se han estado realizando acciones enfocadas en generar alternativas de apoyo para elevar el índice de aprobación, o -lo que es lo mismo- disminuir el índice de reprobación. Estas acciones se llevaron a cabo integrando cuatro áreas de apoyo: el profesor de la asignatura, el tutor del alumno, la coordinación de tutoría y la coordinación de materias propedéuticas.

Para mejorar la eficiencia terminal de los alumnos en esta asignatura, a partir del semestre 2012A -a la fecha-, las acciones se están enfocando en generar alternativas de apoyo, consistentes en: asesorías, talleres, cursos de nivelación y foros. Para este periodo, la materia de Cálculo Vectorial contó con 252 alumnos inscritos, de los cuales $35.71 \%$ eran de segundo curso; del total, 89 solicitaron baja de la asignatura. Considerando que $35 \%$ es un porcentaje alto de bajas, se procedió a la aplicación de un examen diagnóstico ${ }^{4}$ en línea que permitiera detectar las deficiencias que los alumnos tienen en cuanto a conocimientos previos de cálculo se refiere y que fueran necesarios para Cálculo Vectorial, siendo ésta la primera estrategia en aplicar.

Los resultados obtenidos en el examen determinaron las preguntas que presentaron el más alto desconocimiento de temas, los cuales hacen referencia a: determinar la curvatura y torsión de una curva en el espacio, resolver problemas donde las variables independientes están restringidas por el uso del método de multiplicadores de Lagrange para funciones de dos o más variables reales; localizar e identificar los máximos, mínimos y puntos de silla de funciones de dos o más variables reales; y derivar las

4 http://bit.ly/1HxogRU. 
expresiones para determinar un pequeño incremento en una expresión de dos variables reales empleando el Teorema de Taylor; temas que tienen que ser abordados nuevamente por los profesores.

Paralelamente a la aplicación del examen diagnóstico, mediante reuniones con profesores integrantes de la Academia de Cálculo Vectorial, se recabaron opiniones donde atribuyen la poca eficiencia terminal de la asignatura a factores como: "los alumnos no cuentan con bases suficientes de temas de materias antecedentes, los alumnos no dan continuidad a la seriación de las materias debido a la postergación o baja de las asignaturas precedentes dentro del plan flexible de las licenciaturas, existe desinterés del alumno en regularizar su condición, los alumnos no presentan adecuados hábitos de estudio para afrontar la asignatura y la incompatibilidad de horarios de las asesorías y talleres, con relación a los horarios de clases de los estudiantes".

Considerando estas opiniones y los resultados del examen diagnóstico, se reafirmó que una de las causas de un bajo índice de aprobación es que los alumnos no cuentan con los conocimientos previos requeridos para cursar y aprobar de manera satisfactoria la materia de Cálculo Vectorial.

La segunda estrategia fue solicitar a los profesores de la asignatura de Cálculo Vectorial la impartición de algún curso remedial, ${ }^{5}$ de manera que se puedan ofertar los temas con mayor nivel de dificultad en dos diferentes horarios; integrar dentro de la escala de evaluación las asesorías, cuyo desempeño deberá ser avalado por el tutor; encauzar a asesorías a aquellos alumnos que tengan carencias en conocimientos de temas básicos o, bien, si les es posible directamente, proporcionar la asesoría.

Con el claustro de tutores ${ }^{6}$ se implementó la tercera estrategia, haciendo énfasis en la asignatura de Cálculo Vectorial, la cual consistió en: "dar seguimiento más puntual con las actividades académicas del alumno mediante una comunicación semanal por medio del correo electrónico, Facebook, teléfono, entre otras; canalizar de forma directa e inmediata al asesor-profesor(es) del tema solicitado por el alumno; estructurar, junto con el tutorado, un programa de actividades para la Facultad y el hogar, enfatizando la asistencia a las asesorías de Cálculo Vectorial; generar evidencias de las actividades académicas llevadas a cabo por el alumno bajo la tutoría, y orientar al alumno en la toma de decisiones con respecto a su trayectoria académica".

A través de la cuarta estrategia, se implementaron talleres para la resolución de ejercicios. En promedio, se han programado tres talleres por semestre. Estos talleres están enfocados en preparar al alumno para los exámenes departamentales en sus diferentes modalidades (ordinario, extraordinario y título de suficiencia). Cabe mencionar que se ha contado con una asistencia de 15 alumnos en promedio por taller.

Las pláticas de sensibilización e información a través de la Coordinación de Materias Propedéuticas y el Departamento de Tutoría fueron la quinta estrategia implementada, en la cual se entrega a cada alumno un listado de asesorías de Cálculo 1, Cálculo 2, Cálculo 3, Geometría Analí-

5 Curso extracurricular

6 Conjunto de académicos de las diferentes licenciaturas que apoyan a los alumnos en el seguimiento de su trayectoria académica. 
tica y Álgebra, los horarios de asesoría con profesores de la materia y los horarios de talleres de resolución de ejercicios.

\section{Resultados}

Finalmente, se ha llevado un seguimiento de los últimos cuatro años, particularmente se está trabajando con los últimos dos, de los cuales se tiene la información completa de inscripciones y bajas en la referida materia.

El impacto de las acciones y estrategias (asesorías, talleres, cursos de nivelación y foros) fue y sigue siendo significativo, ya que se disminuyó el índice de reprobación de los alumnos de segundo curso, lográndose estabilizar la reprobación entre 10 y $20 \%$ (ver Figura 1).

\section{HISTÓRICO DE REPROBACIÓN DE CÁLCULO 3 DE LOS PERIODOS 20I0A A 20I4A}

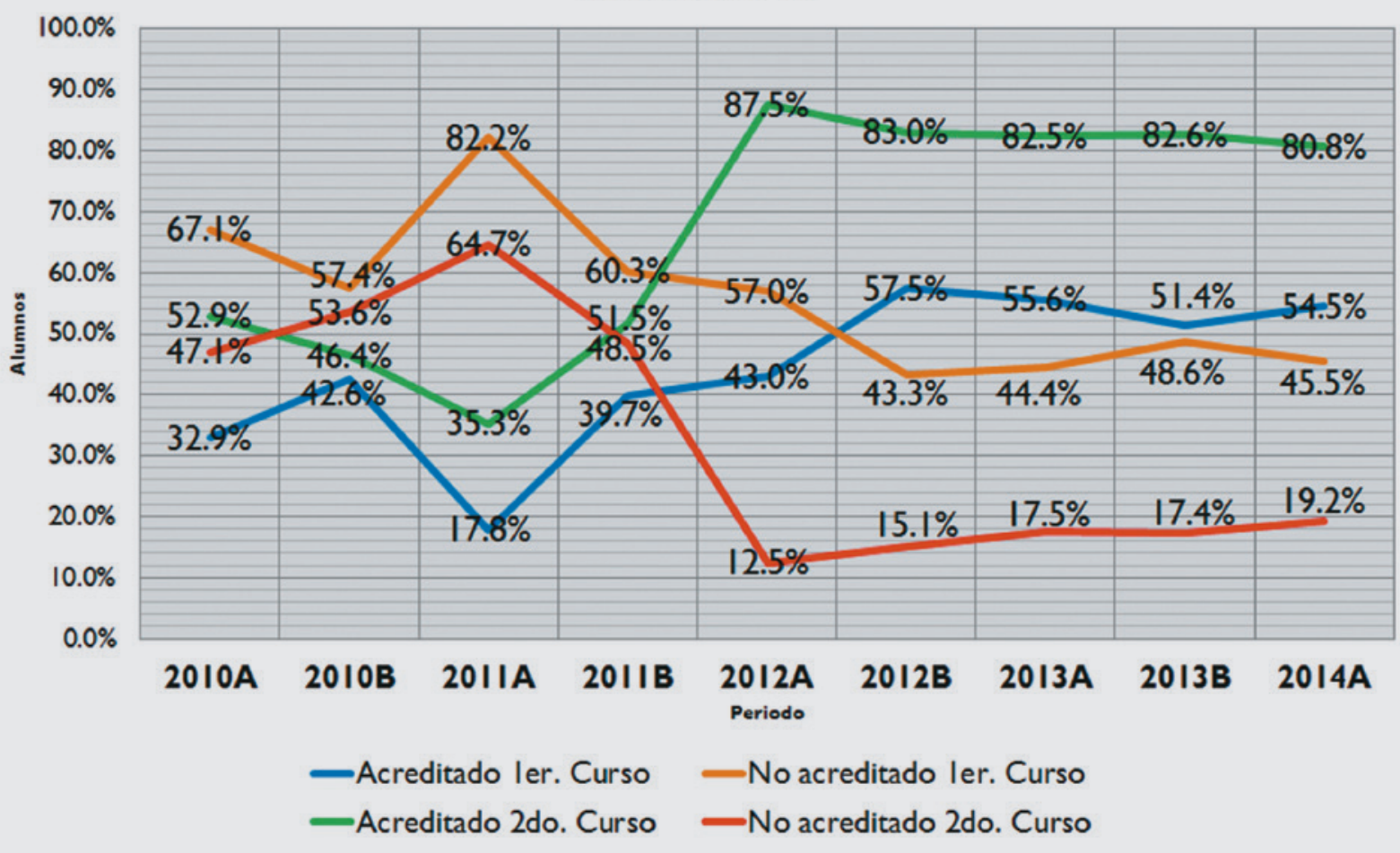

Figura 1. Histórico de reprobación de Cálculo Vectorial.

En la Figura 1, por un lado, se observa que el índice de reprobación disminuyó 36 puntos porcentuales entre los periodos 2011B y 2012A, manteniéndose en un promedio de $80 \%$ la aprobación de los alumnos de $2^{\circ}$ curso y de $16 \%$ en el índice de reprobación, contrastado con $54 \%$ en los periodos anteriores; $y$ por otro, se evidencia un aumento en la permanencia de los alumnos en Cálculo 3, ya que en el período 2010A acreditaron la materia 116 alumnos y en 2014A la acreditaron 201 alumnos (ver Figura 2). 


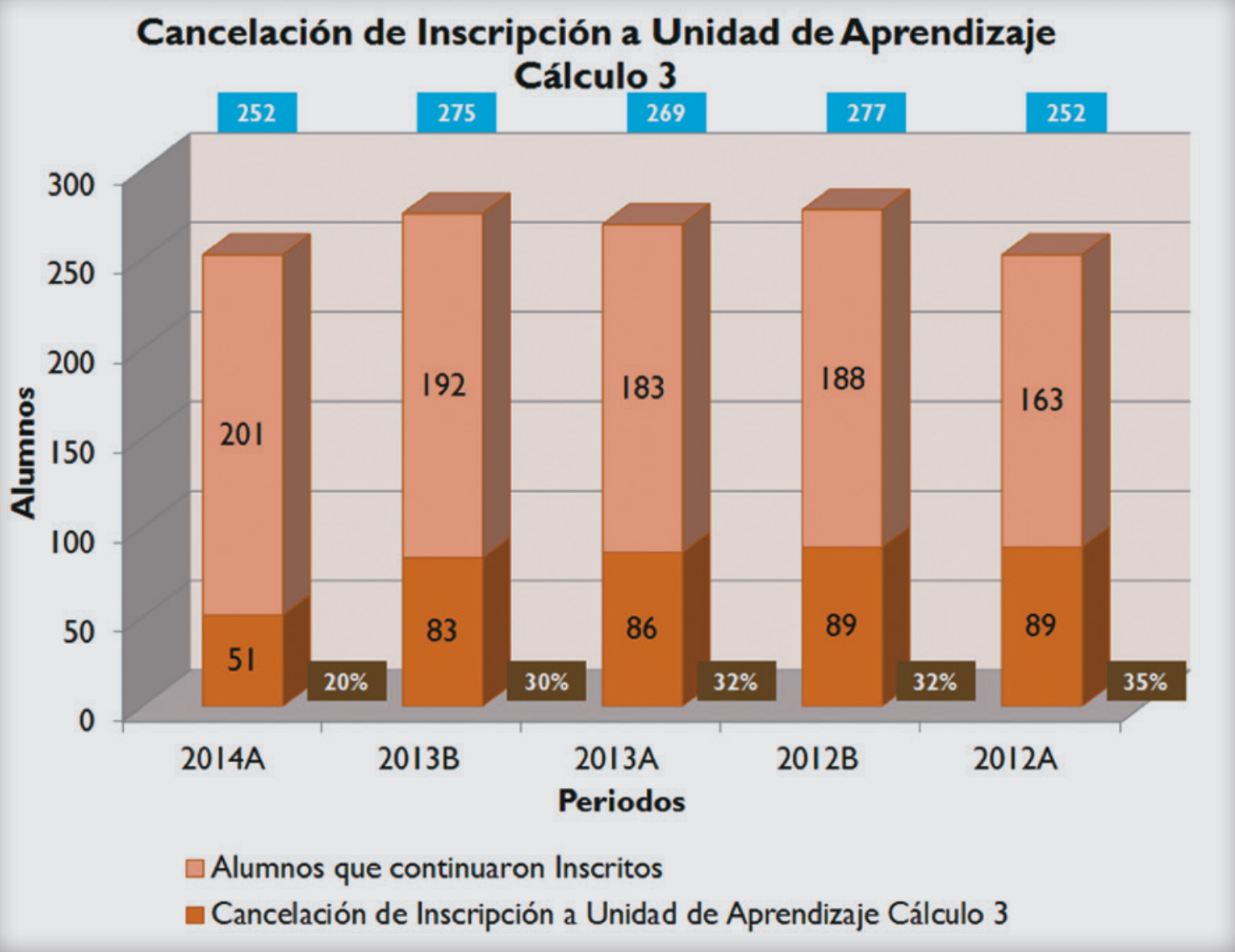

Figura 2. Cancelación de inscripciones de Cálculo Vectorial.

El menor índice de cancelación de inscripción se da en el periodo 2014A; con base en esto, se infiere que podría ser por la sensibilización de los alumnos, ya que se procura hacerlos reflexionar sobre las herramientas y apoyos que la institución ofrece a través de las diferentes áreas.

\section{Conclusiones}

La aplicación de estrategias integradoras para la materia de Cálculo Vectorial logró disminuir el índice de reprobación de $64 \%$ a $12 \%$.

La falta de conocimientos previos es una causa del alto índice de reprobación en la materia de Cálculo Vectorial. Implementar estrategias de apoyo para la acreditación de una materia requiere un trabajo conjunto de diferentes áreas, como la de tutores, profesores, departamentos de apoyo (materias propedéuticas y tutoría) y la de los mismos alumnos.

La aplicación de un examen diagnóstico resaltó la importancia de contar con los conocimientos previos para acreditar una materia consecuente.

Finalmente, debe llevarse a cabo una segunda etapa en la que se contemplará mejorar la eficiencia terminal en los alumnos de primer curso y se comenzará un seguimiento en las materias de Geometría Analítica, Cálculo 2 y Cálculo 1. 
Fuentes de consulta

Aparicio, E. (2004). El cálculo escolar universitario. Un estudio de su problemática en la Facultad de Ciencias. Acta Latinoamericana de Matemática Educativa. México: Universidad Autónoma de Yucatán.

Camarena, G.P. (2008). Teoría de las matemáticas en el contexto de las ciencias. III Coloquio Internacional sobre Enseñanza de las Matemáticas. Perú.

Chávez, M. y Zurita, G. (2005). Estudio estadístico acerca de la deserción estudiantil de la ESPOL: El caso de las Facultades de Ingenierías. Ecuador: Escuela Superior Politécnica del Litoral.

Costa, V.A., Di Domenicantonio, R.M. y Vacchino, M.C. (2010). Material educativo digital como recurso didáctico para el aprendizaje del Cálculo Integral y Vectorial. Unión, Revista Iberoamericana de Educación Matemática, 21, 173-185. Federación Iberoamericana de Sociedades de Educación Matemática.

De La Cruz, J., Sánchez, J. y Urrutia, C. (2008). El proceso de enseñanzaaprendizaje de las matemáticas en ingeniería. 3er. Foro Nacional de Ciencias Básicas, Formación Científica del Ingeniero. México: Universidad Nacional Autónoma de México, Facultad de Ingeniería.

Maplesoft. (2014). Maple. Cybernet Group Company. Recuperado en octubre de 2014 en http://bit.ly/1KU9Fif . Canadá.

MathWorks. (2014). MatLab. MathWorks, Inc. Recuperado en octubre de 2014 en http://www.mathworks.com/.

Montañez, T., González, C., García, M., y Escalante, M. (2009). Cálculo Diferencial con aprendizaje por proyecto empleando Matlab y Robots LEGO NXT. Memorias de la Conferencia Conjunta Iberoamericana sobre Tecnologías para el Aprendizaje. México: Universidad Autónoma de Yucatán.

Ocampo, J., Martínez, A., De las Fuentes, M. y Zataraín, J. (2010). Reprobación y Deserción en la Facultad de Ingeniería Mexicali de la Universidad Autónoma de Baja California. México: Instituto Politécnico Nacional.

Prado, C. y González, L. (2003). Las matemáticas ante el reto de la eficiencia terminal. México: Universidad Nacional Autónoma de México.

Ríos, H.M. (2009). Pre-exámenes como una estrategia didáctica en los cursos de Física. Revista Actualidades Investigativas en Educación. Costa Rica: Universidad de Costa Rica.

Valdez, A., E., Pérez, R., Rodríguez, R., Cubillas, J.M. y Moreno Celaya, I. (2008). ¿Deserción o autoexclusión? Un análisis de las causas de abandono escolar en estudiantes de educación media superior en Sonora, México. Revista Electrónica de Investigación Educativa , 10(1), 1-16. México. 